Ещё одним средством снижения стоимости расхода топлива для самолётов является изменение контура фюзеляжа самолёта. Его днище должно быть не выпуклым, как у современных самолётов, а по большей части плоским, что может создать бо́льшую площадь давления самолёта на воздух. Такое днище будет действовать как ещё одно крыло и помогать крыльям удерживать самолёт в воздухе во время полёта. И, тем самым, снижать расход топлива.

Кроме использования широких, обтекающих корпус фюзеляжа, крыльев, и плоского днища в конструкциях пассажирских и военных самолётов, эти изменения будут весьма полезны и для строительства планеров, существенно удлиняя время их нахождения в воздухе.

Отдавая должное большим заслугам Н.Е.Жуковского в создании теории аэродинамики и строительстве самолётов, следует учесть, что безупречных знаний не бывает, что с развитием науки прежние представления и теории подвергаются уточнениям и правкам. Это касается и теории Н.Е.Жуковского о подъёмной силе крыла самолёта.

$$
* * *
$$

1. Байдаков В.Б., Клумов А.С. Аэродинамика и динамика полета летательных аппаратов. М., 1979. 344 с.

2. Жуковский Н. Е. Теоретические основы воздухоплавания / Н. Е. Жуковский; Научно-Технический Отдел ВСНХ; под ред. В. П. Ветчинкин, Н. Г. Ченцов. - 2- изд. - М. : Гостехиздат, 1925. - 306 с.

3. Карачевский Г.И. Об основной теореме аэродинамики // Воздушно-космическая сфера. 2019. № 4 (101). С. 88-97.

4. Минеев В.Н., Зайдель Р.М. Вязкость воды и ртути при ударном сжатии. ЖЭТФ, 1968. Т.54, №6. C.1633.

5. Платунов Е.С., Самолетов В.А., Буравой С.Е., Прошкин С.С. Физика. Словарь-справочник. СПб: Изд. Политехнического университета. 2018. 798 с.

6. Реджис Эд. Загадка подъёмной силы. Scientific American, 2020, № 4/5. C.164-173.

7. Рыбаков А. П. Особенности фазового превращения воды при ударном сжатии // Прикл. механ. и техн. Сер. Физика. 1996. Т. 37, № 5. С. 17-23.

8. Савиных А.С., Гаркушин Г.В., Канель Г.И., Разоренов С.В. Затвердение воды при динамическом сжатии и его влияние на динамику ударных волн. // Чернышевский сборник, 2017. Т.18, вып.3. С.466474.

9. Drela M. Flight Vehicle Aerodynamics. Mark Drela. MIT Press, 2014.187 p.

10. McLean D. Understanding Aerodynamics: Arguing from the Real Physics. Wiley, 2012.P.251.

\title{
Lin E.E. \\ Asymptotic Method for Studying Kinetics of Formation of Objects with Quantum Features
}

Russian Federal Nuclear Center

(Russia, Sarov)

doi: 10.18411/scienceconf-03-2021-02

idsp: scienceconf-03-2021-02

\section{Abstract}

Asymptotic method for studying kinetics of formation of objects, which reveal quantum features at different space scales has been developed. The method is based on widened interpretation of uncertainty principle and absolute certainty principle and also on conception about object growth from small germs. Proposed phenomenological approach allows us to obtain adequate evaluations of formation characteristics for subnuclear particles and nuclei, crystalline nanoparticles and mesoobjects, for astrophysical/cosmological objects as well. Both principles mutually complement each other and predict formation of objects that so far are not widely known or, at least, well described in scientific literature.

Key Words: asymptotic method, kinetics of formation, object, quantum feature, uncertainty, absolute certainty. 


\section{Introduction}

There are some particular problems in the sciences of microcosm, nano- and mesostructures, astrophysical and cosmological objects. These problems are connected with a determination of spatial boundaries between microphysics and macrophysics as well as of the boundaries of uniformity in matter distribution in the Universe [1-6]. The question arises as to where we can apply ideas about dynamics of quantum systems under the principles of uncertainty [1-3] and certainty $[4,7]$ ?

Object sizes "intermediate" between atoms and solid bodies in mesoscopic crystal structures (which behavior conform to the rules of both quantum mechanics and classical physics) can reach considerable values, at which the lower complexity limit of a macroscopic crystal is reached. Fundamental considerations and assessments of quantum representation feasibility show that the "coordinate-momentum" uncertainty relation can fit for bodies with the sizes about $10^{-7} \mathrm{~m}$, i.e. at a level of nanometer scales. Currently more and more papers deal with investigations of formation, evolution and properties of nanostructures and nanostructure-based materials. These investigations cover processes of various physical and chemical nature proceeding in a mesoscopic region of spatial scales up to $10^{-10}-10^{-4} \mathrm{~m}$ : from formation of atomic clusters on a molecular level and nanocrystal synthesis to global changes of a macroscopic continuum state [8-13]. Mechanical behavior of nanostructured objects is described using molecular dynamics methods and using statistic models allowing for both thermodynamic and kinetic aspects of problems under consideration. These problems cover incompleteness of classical description of process running in mesoscopic scales, necessity of developing new models including phenomenological ones that take into account formation of quantum systems and determine growth regularities and nanostructure consolidation, and determination of phase transformation size dependencies, in particular, ascertainment of the effect of polymorphic transformations on synergy of crystal structures.

In this connection it is essential to determine general features of dynamics of object growth both in microcosm and in "mesocosm". With this purpose this work investigates kinetics of formation of various in physical nature compact objects having strong internal bonds and considerably different spatial scales: nuclear matter clusters [14] and mesoscopic structures with covalent and quasi-covalent bonds between atoms in a nanocrystalline lattice [15]. A typical feature of these objects is existence of purely quantum effect of exchange interaction. Virtual meson exchange occurs between nucleons, electron exchange occurs in crystal structures having covalent bonds. The effect of exchange interaction allows these objects to be viewed as compact clusters with pronounced collective quantum properties. In case of nuclei these quantum properties are connected with strong interactions and manifest themselves in the existence of oscillatory and rotatory shells. In mesocrystals quasi-particles (phonons) are excited.

\section{Brief Review of Achieved State of Notions}

The above creates the necessary precondition for investigating kinetics of object formation from a common point of view. We consider closed stochastic systems of compact quantum clusters randomly interacting with each other. In accordance with standard ideas of physical kinetics the irreversible aggregation of objects is described using the concept of distribution density wave $\varphi(a, t)$ propagating with the time $t$ in the space of cluster sizes $a$ in direction of these growth. It is noted in [16] that the behavior of a wave packet of any physical nature is subjected to the universal ratio for half-breadth of a wave packet and halfbreadth of a spectral line $\Delta a \cdot \Delta k \geq 1 / 4 \pi$ ( $k$ is wave number), which is afforded by the Fourier theorem. One can assume that this circumstance results in the uncertainty relation for coordinate and momentum in the space of cluster sizes. For quantum-mechanical systems being in a coherent state (harmonic oscillations), the above product takes the minimum value, the uncertainty relation is fulfilled as an accurate equality, and process trajectory in phase space is classical [17]. This means it is possible to consider relatively large objects which sizes exceed values corresponding to the lower limit of complexity for a macroscopic solid. 
Thus, the proposed phenomenological approach describes a limiting asymptotic stage of cluster growth process. Under this approach "the leading" processes appear to be oscillations of components that compose objects, for example collective oscillations of nucleons in a nucleus or phonon excitations of mesostructure crystal lattice.

It should be noted that the proposed asymptotic method in no way can replace the developed fundamental methods of investigations and physical models of microcosm and mesocosm dynamics described in the above Ref. [1-10] and represented in [18-21]. On the other hand it is well known that when interacting with environment, physical objects never discover their complete internal complexity potential (see [2]). Hence incomplete or even phenomenological description of physical phenomena or physical objects sometimes responses better to the main point, and to the understanding of what is going on.

For example, when solving kinetic equations the fitting parameters determined using experiments, are introduced, as a rule. When planning experiments with new or weakly studied objects, there appears necessity to perform preliminary evaluations of final dimensional and timing characteristics of the investigated processes, i.e. to determine dependence between typical object sizes and the time of their growth. In this respect, the proposed asymptotic models can be useful when first meeting this or that problem, evaluating it or analyzing primary experimental data. For example, when studying cluster radioactivity described in [22], this method made it possible to determine the spectrum of the most probable mass numbers of relatively light nucleon clusters formed inside heavy nuclei [14]. This method also makes it possible to evaluate both average and maximum mass numbers of super-heavy nuclei, formed inside supernovas and to determine mass numbers corresponding to the "peaks" of abundance of elements in Galaxy, numerically computed in [23]. In addition, it turned out to be possible to get estimates of time formation and typical sizes of neutron stars at supernova outbursts. In the field of mesoscopics this approach is valid and reasonably effective for materials with profound strong interatomic bonds: carbon nanostructures with covalent bonds [15] as well as for nanostructured particles of biological objects [24].

One of topical trends of nanoscience and nanotechnology consists in the creation and the study of biological materials, in particular, the study of physical mechanisms of protein biosynthesis $[5,12,25]$. In accordance with the results of the investigations the commonly accepted scheme of proteins construction is presented in such a manner that a volumetricpacked nanoparticle that represents an aperiodic crystal, is formed from nanochains with the $\mathrm{C}-\mathrm{N}$ peptide bonds (primary structures) as a result of the twisting and the mutual arrangement of various polypeptides in the presence of nucleic acid molecules.

Besides, in [26] the possibility exists «that the given scheme does not exhaust all ways of a biosynthesis of proteins». In the light of this the quantum nature of biophysical processes should be noted, because thanks to the quantum nature «the main peculiarities of physical behavior of macromolecules are determined by rotational isomerism», because a substance is considered as a dynamic mixture of amino acid molecules located in crossed conformation and in conformations directed to the right and to the left [25]. Hundreds and thousands of vibrations with the frequencies of the order of $10^{12}-10^{13} \mathrm{c}^{-1}$ occur in a turning time of the order $10^{-10} \mathrm{~s}$ [25] in a molecule. In the course of these vibrations the bonds can be formed between contiguous molecules - tunnel transition takes place in a quantum-mechanical system as a result of a great number of «attempts». Hence, rotational-vibrational interactions of amino acid molecules can bring about their volumetric polycondensation. Inoculating centers of polycondensation can be nucleic acid molecules, close to which amino acid molecules are clustered in a certain order, prescribed by a preferable formation of $\mathrm{C}-\mathrm{N}$ bonds in the system volume, as the shortest and strongest bonds in comparison with $\mathrm{C}-\mathrm{C}$ bonds and $\mathrm{C}-\mathrm{O}$ bonds (see [27]).

In work [24], on the basis of the asymptotic method of [28] for studying kinetics of formation of objects having quantum features, a scheme of a direct synthesis of volumetric 
protein nanoparticles is considered in a system previously consisted of amino acid molecules with additives of nucleic acid molecules (cytoplasm [5]). A protein synthesis without a formation stage of polypeptide nanochains is seemingly possible owing to phonon excitations of molecules in the whole volume. It is demonstrated in work [28] that the mentioned method is also used in a mesoscopic range of sizes. This makes it possible to use this method to assess a maximum size of biological objects under consideration - cells. Thus, the wisdom of pursuing development of asymptotic models [28] in the field of mesoscopics is connected with both creation of ultra-hard carbon materials and synthesis of biological materials.

At present the increasing number of scientific investigations is devoted to different problems of astrophysics and cosmology: from formation and evolution of stars to formation of the large-scale structure of the Universe under influences of dark matter and dark energy (see, for example, [1, 6, 29]). The results of these investigations are based on reliable astronomic observations as well as on modern theoretical conceptions about formation and expansion of the Universe after Big bang. Theoretical studies are connected with the ideas on behavior of gravitational fields and gravitating masses at diversiform possible geometries of cosmic space, in particular, at Euclidean, parabolic and hyperbolic geometries. Stochastic quantum nature of cosmological phenomena is noted.

The most significant cosmological problem of $[1,6,29]$ is determination of spatial boundaries corresponded to uniformity and isotropy of the Universe, i.e., to Cosmological principle. The latter signifies that because of insufficient time for growth of gravitational perturbations, the distribution of matter at early stages of formation of cosmological structures does not affect the average value of matter density in up-to-date cosmos, and "heavy heterogeneities and voids are ended at some very large but still unknown distance"]. Another significant problem is the following: is cosmos infinite or finite (closed) and where is a boundary of closed cosmic space?

In accordance with conception of [6] about a large-scale structure of the Universe as of non-uniform matter distribution originating from growing adiabatic perturbations of density, there is some distinction between the developed non-linear structures within the scales of less than 10 Megaparsec (Mps) (namely, halo of the galaxy, groups and clusters), and the structures with more regular quasi-linear matter distribution within the scales of up to 100 Mps, i.e. superclusters and cosmological "voids". It should be noted that at present the quasiundisturbed Hubble flow of matter that has been already affected by quantum-gravitational instabilities, still continues.

In the paper [30] an attempt of qualitative analysis of the above cosmological problems is undertaken. With this purpose extended interpretation of an uncertainty principle applied to the sizes of objects in cosmic scales is proposed. The proposed analytical approach makes it possible to obtain asymptotic time dependences for characteristic sizes of astrophysical and cosmological objects in approximation of spherical symmetry in Euclidean geometry. Globular star clusters, superclusters of Galaxy and the Universe itself are considered as such objects. The existence of groups of interacting universes is postulated, and the issue of a size of cosmic sphere involving the great number of such groups is considered.

2. Revealing the Uncertainty Principle and Absolute Certainty Principle in

\section{the Kinetics of Objects Formation}

Phenomenological growth laws [31] of volume packed object sizes with the time $t$ and expressions for their characteristic sizes in formats of the uncertainty and absolute certainty relation within the space of sizes $a$ are given in Table 1 .

Table1

Relationships, laws of growth and formulae for characteristic object sizes

\begin{tabular}{|c|c|c|}
\hline Objects & Uncertainty principles $(u n)$ & Absolute certainty principles $(a c)$ \\
\hline Microcosm and & Relationships & Relationships \\
mesostructures & $\Delta a \Delta p \approx \hbar / 2$ & $a p=\hbar / 2$ \\
& $\Delta t \approx \hbar / \Delta E$ & $E t=\hbar$ \\
\hline
\end{tabular}




\begin{tabular}{|c|c|c|}
\hline & $\begin{array}{c}\text { Growth laws } \\
\langle a\rangle^{3 / 2} d\langle a\rangle \approx\left(\frac{\hbar a_{0}^{3}}{2 m_{0} t_{i}}\right)^{1 / 2} d t \\
\langle a\rangle^{3 / 2} d\langle a\rangle \approx\left(\frac{a_{0}^{3} \Delta E}{2 m_{0}}\right)^{1 / 2} d t \\
\text { Maximal size } \\
a_{\max }^{\text {un }} \approx \frac{2 m_{0} a_{0}^{3}}{9 \hbar \Delta t_{\min }}\end{array}$ & $\begin{array}{c}\text { Growth law } \\
a^{4} d a=(\hbar / 2 \alpha \rho) d t \\
\text { Characteristic size } \\
a_{c h a r}^{a c} \approx\left(\frac{3 \hbar t_{i}^{\text {char }}}{2 m_{0}}\right)^{1 / 2}\end{array}$ \\
\hline $\begin{array}{l}\text { Objects of } \\
\text { astrophysics and } \\
\text { cosmology }\end{array}$ & $\begin{array}{c}\text { Relationships and growth law } \\
\qquad|\Delta a| \cdot|\Delta p| \cong K_{c} / 2 \\
|\Delta E| \cdot|\Delta t| \cong K_{c} \\
\langle a\rangle^{2} d\langle a\rangle=(1 / \sqrt{2})\left(\rho_{c} / \rho\right)^{1 / 2} a_{0}^{2} c d t\end{array}$ & $\begin{array}{l}\text { Relationships and growth law } \\
\qquad a p \cong K_{c} / 2 \\
E t \cong K_{c} \\
\langle a\rangle^{2} d\langle a\rangle=(1 / 2)\left(\rho_{c} / \rho\right) a_{0}^{2} c d t\end{array}$ \\
\hline
\end{tabular}

Here $\Delta p \sim p=m \Delta a / \Delta t$ is uncertainty of momentum $p, m$ is object mass, $\Delta E$ is width of energy level $E$ of the excited state of quantum mechanical system determined by nature of objects and a mode of process, $\hbar$ is a reduced Planck constant, $a_{0}, m_{0}$ is size and mass of an embryo, $t_{i}$ is characteristic time scale of an elementary (single) act of objects interaction, $\alpha$ is geometrical factor (for a cube $\alpha=1$, for a sphere $\alpha=\pi / 6$ ), $K_{c}$ is phenomenological action constant in cosmic scales, $\rho$ is density of observed substance in the Universe, $\rho_{c}$ is substance critical density at which the Universe becomes closed, $c$ is light velocity. Physical meaning of the relationship between uncertainties "coordinate - momentum" is in the fact that during a period of time $\Delta t \equiv t_{i}$ of elementary (single) act of objects interaction, the exact size of each object cannot be determined until this interaction is finished. It is associated with the fact that up to the end of the single act, it is impossible to determine the correlation between the object and each interacting surface element. In the format of absolute certainty, the relationship "coordinate - momentum" implies that at each time, the object under consideration is strictly localized within the space of sizes.

Table 2 provides examples of how the above principles manifest themselves when describing formation of objects in the process of equilibrium approximation. The given results are in agreement with the generally known conceptions. One can see that under the proposed kinetic approach the uncertainty principle covers a wider spectrum of processes for objects formation as compared to the absolute certainty principle. At the same time, both principles mutually complement each other. Thereby, the developed asymptotic method for investigating the kinetics of formation of objects with quantum properties, corresponding to the statement [2] about adequacy of phenomenological description of physical phenomena or physical objects, has a sufficient level of generality to be used in problems of high energy density physics and in physical chemistry of high intensity processes. Below are the examples of how uncertainty principles and absolute certainty principles are applied in problems of objects formation in microcosm, nano- and mesocosm, and cosmos.

Table 2

Manifestation of principles in the process of equilibrium approximation. Examples.

\begin{tabular}{|c|l|l|}
\hline Objects & \multicolumn{1}{|c|}{ Uncertainty principle } & Absolute certainty principle \\
\hline Microcosm & $\begin{array}{l}\text { Formation of hadron jets from } \\
\text { quarks } \\
\text { r-processes; stable nuclei } \\
\text { Deep inelastic relativistic processes }\end{array}$ & Formation of groton from quarks \\
\hline
\end{tabular}




\begin{tabular}{|c|c|c|}
\hline & $\begin{array}{l}\text { in nuclei } \\
\text { Formation of super-heavy nuclei up } \\
\text { to mass number } A_{\text {end }} \cong 470\end{array}$ & $a_{\max }^{a c} \approx 4.8 \times 10^{-12} \mathrm{~m}$ \\
\hline $\begin{array}{c}\text { Carbon nanoparticles and } \\
\text { mesoobjects }\end{array}$ & $\begin{array}{l}\text { Formation of all known artificial } \\
\text { and natural diamonds with sizes } \\
\text { from } 0.7 \mathrm{~nm} \text { to } 20 \mathrm{sm} \\
\text { Characteristic sizes of protein } \\
\text { nanoparticles and mesoobjects } \\
\text { (archaea, cells) }\end{array}$ & $\begin{array}{l}\text { Characteristic size of a carbonado- } \\
\text { type diamond }(\approx 0.1 \mathrm{~mm}) \\
\text { Characteristic size of insulin }\end{array}$ \\
\hline Stars & $\begin{array}{l}\text { Characteristic times of formation } \\
\text { and a size of neutron stars: } \\
0.17-17 \mathrm{~s}, 16 \mathrm{~km}\end{array}$ & $\begin{array}{l}\text { Characteristic size of dwarfs } \\
\approx 4000 \mathrm{~km}\end{array}$ \\
\hline Superclusters of galaxies & 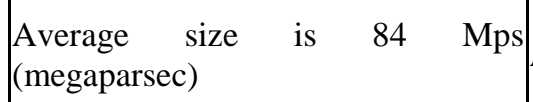 & Average size is $36 \mathrm{Mps}$ \\
\hline
\end{tabular}

\subsection{Microcosm}

Based on the law of growth for objects in the format of the uncertainty principle [28], it is possible to evaluate the value of fundamental mass in microcosm $m_{\text {fund }}$, if one accepts that the least spatial unity (fundamental length) is the value $a_{f u n d} \approx 10^{-18} \mathrm{~m}$, and that this value is matched by the time scale $t_{u n}=a_{\text {fund }} / c$. In [28] the following equation for fundamental mass is given:

$$
m_{\text {fund }}=\frac{\hbar}{c a_{\text {fund }}} .
$$

It follows from here that $m_{f u n d} c^{2}=\hbar c / a_{\text {fund }}=196 \mathrm{GeV}$ [28]. The obtained value approximately corresponds to the mass value of a dark matter particle determined in [32] according to the data of astrophysical observations and equal to $192 \mathrm{GeV}$. It should be noted that expression (1) is definitely connected with determination of Compton wave-length of a material particle $\lambda=\hbar / m c$, and the above value of a fundamental length is adequate to the current concepts.

At format of absolute certainty principle one can obtain from the relation $a_{\text {char }}^{a c} \approx\left(\frac{3 \hbar \Delta t_{\text {char }}}{2 m_{0}}\right)^{1 / 2}$ that the size of hadron formed from light $u$-quarks and $d$-quarks with rest mass $330 \mathrm{MeV}$ [19] in confinement station $\left(m_{0}=0.58 \times 10^{-27} \mathrm{~kg}\right)$ and at $\Delta t_{\text {char }}=10^{-23} \mathrm{~s}$ (time scale of strong interaction [33]) is equal to $a_{\text {hadr }} \approx 1.64 \mathrm{Fm}$. Obtained value is in good agreement with the "electrical" diameter of proton which is equaled to $1.72 \mathrm{Fm}$ [33]. Thus, space characteristic of nucleon is based on mass characteristic of quark without size (conditional "point" having the mass). If we consider the "obtained" nucleon with rest mass $m_{0}=1,67 \times 10^{-27} \mathrm{~kg}$ as the germ, then it follows from the above formula that at characteristic nuclear time $\Delta t_{\text {char }}=10^{-21} \mathrm{~s}$ nuclear size equals to $a_{\text {nucl }} \approx 9.7 \mathrm{Fm}$. Mass number of such nucleus is equal to $A_{n u c l}=58$ (nuclear radius $R=r_{0} A^{1 / 3}, r_{0} \approx 1.25 \mathrm{Fm}$ [34]). The obtained value correspond to interval of mass numbers near "iron peak" of elements spreading in Universe [33]. These results supplement with notions of work [7] about the role of certainty in microphysics.

General diagram (Figure 1) of stable neutron-rich nuclei has been obtained in work [34] with regard for uncertainty principle. 


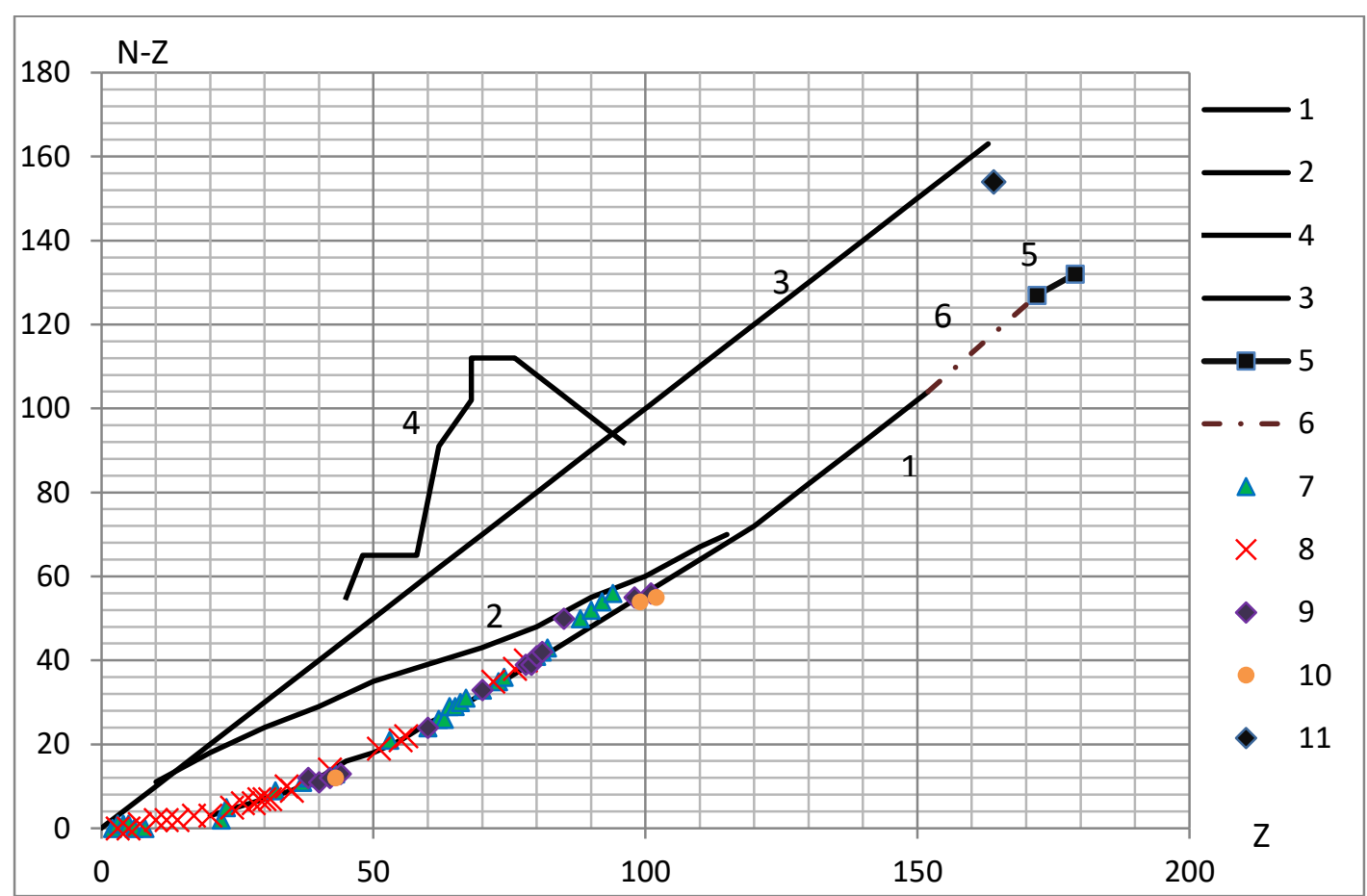

Figure 1. Diagram of neutron-rich nuclei:

1 - parabolic line of $2 \beta$-stability [35] regarding for experimental data with prolongation up to hypothetical final nuclide $Z=152, N=258 ; 2$ - the boundary of well-known neutron-rich nuclei [33]; 3 - supposed boundary of the existence of neutron-rich nuclei (tritium medium); 4 -the boundary of neutron stability [36]; 5 - final nuclides $Z=172, N=299$ u $Z=179, N=311$ [14, 28, 34]; 6 -extrapolation of line 1; 7 - elements calculated jointly with Weizsacker formula of $\beta$-stability [33]; 8 - stable isotopes calculated jointly with Weizsacker formula; 9 - elements calculated jointly with nuclei systematics [35]; 10 - isotopes calculated jointly with systematics [35]; 11 - "island" of stability $Z=164, N=318$ mentioned in [35].

One can see that the calculations performed by use of asymptotic method [34] correspond to parabolic line of $2 \beta$-stability [35], and to notions of monograph [33]. Point of intersection of lines 3 and 4 correspond to hypothetical stable exotic nucleus ${ }^{281} \mathrm{X}_{93}$. In addition, the boundary 3 lies not far from the "island" of stability $Z=164, N=318$ mentioned in [35].

\subsection{Nanometric and mesoscopic objects}

In the field of nanosized scale and mesoscopics the uncertainty principle covers the whole spectrum of object formation processes described in [5, 12, 37, 38]. Two types of objects are considered: 1) clusters with the lattice formed of atoms of a single type, oscillating as harmonic oscillators with the typical frequencies $\sim 10^{12}-10^{14} s^{-1}$ ("atomic" nanocrystals); 2) clusters formed by macromolecules revealing both oscillating nature of inner motions with the given frequencies, and rotational isomerism with the frequencies $\sim 10^{10}-10^{11} \mathrm{~s}^{-1}$ ("molecular" nanocrystals). The question arises of whether there is any influence of collective quantum structure properties on the processes of their formation and growth and on the values of their typical sizes. As the objects of the first type, it is reasonable to consider crystals with covalent carbon bonds $\mathrm{C}-\mathrm{C}$ i.e., nanodiamonds characterized by expressed phonon effects associated with exchange interaction of atoms. As the objects of the second type, it is reasonable to consider protein nanoparticles consisting of amino acid molecules, since the latter are characterized by strong bonds of $\mathrm{C}-\mathrm{C}, \mathrm{C}-\mathrm{N}$ and $\mathrm{C}-\mathrm{O}$ type that provide highfrequency oscillating constituent of internal motion, and by rotational isomerism and lowfrequency component of spin-lattice relaxation creating conformational motion with the typical times $\tau=10^{-10}-10^{-7} \mathrm{~s}$.

In general, the mechanism of formation of macroscopic diamond particles from nanodiamonds described in $[15,28,34]$ covers all available data about sizes relating to both artificial diamonds obtained in static and dynamic synthesis, and natural diamonds. 
One of the important trends in nanoscience and nanotechnology is formation and investigation of biological materials, in particular, the study into physical mechanisms of protein biosynthesis $[5,12]$. According to investigation results, the agreed system of protein arrangement looks like the following: out of nanochains with peptide $\mathrm{C}-\mathrm{N}$ bonds (primary structures) as a result of twisting and relative position of various polypeptides, in the presence of molecules of nucleic acid, a volume-packed nanoparticle that represents an aperiodic crystal with the given biological code is formed.

At the same time, in [24, 34], there is another system of arrangement of biological nanoparticles, in which as a result of rotational-vibrational interaction of amino acid molecules, their volumetric polycondensation can occur. The seeding centers of polycondensation can be the molecules of nucleic acids, around which amino acid molecules are grouped. They are grouped in a certain order given by preferable formation of $\mathrm{C}-\mathrm{N}$ bonds as the most short and strong bonds as compared to $\mathrm{C}-\mathrm{C}$ and $\mathrm{C}-\mathrm{O}$ bonds. In the proposed in $[24,34]$ way of considering the corresponding mechanism of protein nanoparticle synthesis, the principle of uncertainty assumes the possibility of mutation of biological objects at a molecular level. Based on typical sizes and masses of embryos (molecules of amino acids of glycine, alanine, valine and tryptophan), there have been determined the following vicinities of the most probable sizes and "magic" sizes, corresponding to proteins [5, 12, 38]: (1.4-1.7); (2.2-2.5); (2.7-2.9-3.3); (4-4.6-4.7); (5-5.5-5.6); (6-6.7-7); (8-8.5); 9; 10; 11; 12; 14; 15; $16 ; 17 ; 18 ; 19 ; 20 ; 21 ; 22 ; 23 \mathrm{~nm}$. The calculated maximum sizes of mesoobjects from above listed embryos provided the values $0.63 ; 1.1 ; 2.1$ and $7 \mu \mathrm{m}$, corresponding to lysosomes, mitochondrions, red cells, thrombocytes, and small lymphocytes. The calculated results testify to the fact that at the "instant" excitation of a biological system, e.g., under absorption of the radiation energy of various nature, it is possible that nanoparticle and mesoobject significantly increase in sizes, and low density lipoproteins and leucocytes are formed. This fact does not contradict to the known medical facts of formation of mutations and tumors or development of atherosclerosis and leukemia under the effect of ray penetration into an organism.

In case of continuous protein nanofibres (linear nanostructure), one can use the method from [24] and obtain the following expressions for determining typical values of the thickness $d$ and the length $l$ of the objects:

$$
\begin{gathered}
d=a_{0}\left(\frac{2 m_{0} a_{0}^{2}}{\hbar t_{i}}\right)^{1 / 4}, \\
\langle l\rangle=\left(\frac{\hbar}{m_{0}} t\right)^{1 / 2} .
\end{gathered}
$$

With the typical parameter $t_{i} \equiv t_{R}=2 \pi \hbar b / k_{B} \sqrt{2 \theta_{R} T}$ [39] corresponding to rotational molecule isomerism ( $k_{B}$ is Boltzmann constant, $b$ is a number of crystal-forming bonds, $\theta_{R}$ is the typical rotational bond temperature $(\approx 2.6 \mathrm{~K}), T$ is ambient temperature), we derive from the formula (2) that for the embryos like glycine (the least amino acid with $m_{0}=1.25 \cdot 10^{-25} \mathrm{~kg}, a_{0}=0.67 \mathrm{~nm}$ [5]) and tryptophan (the highest amino acid with $m_{0}=3.4 \cdot 10^{-25}$ $\left.\mathrm{kg}, a_{0}=0.67 \mathrm{~nm}[5]\right)$ at $T \cong 310 \mathrm{~K}$, the diameters of nanofibres are equal to $1.27 \mathrm{~nm}$ and 2.35 $\mathrm{nm}$. These values approximately correspond to the thicknesses of collagen protein $(\sim 1 \mathrm{~nm}[5])$

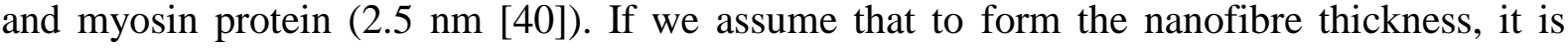
sufficient to have a single oscillation of a "last bond" of the longest molecule of tryptophan embryo and $t_{i} \cong 3 \cdot 10^{-14} \mathrm{~s}$ [9], then from formula (2) we get $d \cong 11.6 \mathrm{~nm}$. This approximately corresponds to the thickness of a continuous neurofibril of a human, neurofilament, approximately equal to $10 \mathrm{~nm}$ [41]. Calculation according to formula (3) shows that to form a neurofilament $300 \mathrm{~nm}$ long, one requires approximately $100 \mu \mathrm{s}$.

Thereby, the results from the relationship "coordinate-momentum" in the space of object sizes are indicative of the fact that in the system of amino acid molecules, accidental formation of quasi-crystalline nanoparticles and mesoobjects corresponding in their sizes to 
essential proteins and cells is possible. These "incorrect" (mutational) objects can grow on these or those crystallization centers without formation of polypeptide bonds, i.e., without formation of "correct" biological code. At the same time formation and growth of such nanoparticles and mesoobjects is possible on fragments of damaged proteins and cells as on the centers of crystallization. All this is in compliance with the generally known concepts concerning mutations of biological structures at a molecular level.

As for the all-known ideas concerning possible origin of life on the Earth as a result of amino acids brought onto the Earth from space, in [24, 34], in the format of the uncertainty principle, it is shown that the objects with sizes from 30-45 nm (ribosomes, inside which protein synthesis occurs) to $0.4 \mu \mathrm{m}$ (nanosized symbiont) can be generated from amino acid fragments formed under impacts of meteorites against the earth surface.

\subsection{Astrophysics and cosmology}

With reference to the processes of cosmic scale, it should be noted that the uncertainty principle [30] predetermines currently observed Universe accelerated expansion described by de Sitter cosmological model and Hubble law [1]. In addition the estimated radius of a cosmic sphere $R_{c} \cong 4.5 \cdot 10^{27} \mathrm{~m}$ including the great number of independent from each other groups of universes that interact between themselves inside each group within a "light" sphere with the radius $R_{\text {light }} \cong 1.323 \cdot 10^{26} \mathrm{~m}$, reasonably corresponds to a cosmic radius $R_{c B} \cong 5.89 \cdot 10^{27} \mathrm{~m}$, determined in work [42] based on relationship between physical constants. The existence of multiple interacting universes does not contradict the statement about existence of a set of images of a "unique specimen" of [42].

One can try to evaluate the range of "rigid' sizes of astrophysical and cosmological objects based on the principle of absolute certainty. In case of supernova explosion, one should substitute Plank constant in the proper formula of Table 1 by the determined in [28, 34] phenomenological action constant in the "World" of collapsing stars equal to $K_{G}=\mathrm{M}_{C h}^{2} G_{N} / c=1.72 \cdot 10^{42} \mathrm{~J} \times \mathrm{S}\left(G_{N}\right.$ is a gravitation constant, $\mathrm{M}_{C h}$ is Chandrasekhar critical mass starting from which a star begins to collapse):

$$
a_{\max }^{a c}=\hbar\left(\frac{3}{2 m_{0} E_{\min }}\right)^{1 / 2} \rightarrow a_{\max }^{a s t r}=K_{G}\left(\frac{3}{2 m_{0} E_{\min }}\right)^{1 / 2}
$$

The minimum value of the carried away energy at supernova explosion is equal to $E_{\min }=10^{41} \mathrm{~J}$ [43]. As a seed mass, we will formally accept Chandrasekhar limit $m_{0}=\mathrm{M}_{C h} \cong 1.4 \mathrm{M}_{\text {Sol }}, \mathrm{M}_{\text {Sol }}$ is the mass of Sun [44]. Hence, we obtain that $a_{\text {max }}^{a s t r} \cong 4 \times 10^{6} \mathrm{~m}$ that approximately corresponds to the size of dwarfs [44]. To determine the maximum possible cosmological size, one should substitute into the proper formula $a_{\max }^{a c}=\left(3 K_{c} t_{i}^{\max } / 2 m_{0}\right)^{1 / 2}$ the limit value of the cosmological action constant determined in $[30,34]$ as $K_{c}^{\lim }=M_{c}^{2} G_{N} / c$. Here, $M_{c}=m_{p} N_{b}\left(\rho_{c} / \rho\right) \cong 5.5 \cdot 10^{54} \mathrm{~kg}$ is cosmic mass determined as a product of baryon mass and the ratio between critical density and baryon density, $m_{p}$ is proton mass, $N_{b} \approx 10^{80}$ is baryon number in the Universe [1]. We will accept the value $M_{c}$ as embryo mass. Then with $t_{i}^{\max }=a / c$ we obtain the expression for a "new' phenomenological constant that determines the value of the maximum cosmological size $a_{\max }^{c}$ in the format of the absolute certainty:

$$
a_{\text {max }}^{c}=\frac{3 M_{c} G_{N}}{2 c^{2}} \cong 6 \times 10^{27} \mathrm{~m} .
$$

This value are in correspondence with the size for cosmic sphere of $9 \times 10^{27} \mathrm{~m}$ calculated in [30] with regard for uncertainty principle.

\section{Issues for Further Studies}

In terms of evolution of the results, one can try to consider the possibility of formation of objects that have not been yet discovered or are not widely known and therefore, are not described in scientific literature in details. 
3.1. In microcosm in the format of the uncertainty principle, such an object can be a final nuclide with a mass number near to $A_{\text {end }} \cong 470[14,28,34]$. In a format of the absolute certainty, with the help of formula $a_{c h a r}{ }_{c a} \approx\left(\frac{3 \hbar t_{i}^{c h a r}}{2 m_{0}}\right)^{1 / 2}$ in the case of alpha-particles as germs and at $t_{i}^{\text {char }}=10^{-15} \mathrm{~s}$ (lifetime of intermediate composite nucleus), one can assume an existence of giant nuclei with the size $a_{\max }^{a c} \approx 4.8 \times 10^{-12} \mathrm{~m}$. The evaluated size of a hypothetical giant nucleus is in an order of magnitude a thousand times greater than the spatial scale $\sim 10^{-15}$ $\mathrm{m}$ of strong interaction between nucleons in usual nuclei. Due to Coulomb repulsion of protons, nuclear forces cannot hold compactly such a huge system consisting of protons and neutrons bound by just strong interaction. It should be noted that the calculated nucleus size corresponds in an order of magnitude to the Compton wavelength of muon neutrino/antineutrino with the rest mass $m_{v_{\mu}}=0.19 \mathrm{MeV}$ [33]: $\lambda=\hbar / c m_{v_{\mu}}=1.05 \times 10^{-12} \mathrm{~m}$. It is reasonably to assume that inside such nucleus under the effect of muon antineutrino, a wellknown reaction [33] takes place, i.e., disintegration of a proton into neutron and a positively charged muon:

$$
\bar{v}_{\mu}+p \rightarrow n+\mu^{+} .
$$

This reaction assumes formation of "neutron" nuclei in material domains rich in muon antineutrino. The latter are particles "gluing" giant nuclei from inside similarly to what pions do in usual nuclei [33]. Apparently, such hypothetical nuclei can be near to neutron star surfaces (in a crust or liquid domain), where heavy nuclei are located [33, 44].

The abovementioned similarity of muon antineutrino and pions implementing the strong interaction between nucleons in nuclei, allows us to put a question whether muon antineutrino are carriers of some type of interaction between nucleons inside giant nuclei similar to strong interaction. Table 3 provides comparison of characteristics of these two types of interaction. One can see that Yukawa potentials inside the considered nuclei are comparable in an order of magnitude. Right this makes the existence of hypothetical giant nuclei possible.

Table 3

Interaction capabilities between nucleons inside nuclei.

\begin{tabular}{|c|c|c|}
\hline Nuclei & Usual & Giant \\
\hline Type of interaction & Strong & Assumed \\
\hline Carriers & Pions & Muon antineutrino \\
\hline Rest mass $m_{r}, \mathrm{MeV}$ & 139.57 & 0.19 \\
\hline $\begin{array}{c}\text { Compton wavelength } \lambda, \mathrm{m} \\
\lambda=\hbar / m_{r} c\end{array}$ & $1.46 \times 10^{-15}$ & $1.05 \times 10^{-12}$ \\
\hline Nucleus radius $R, \mathrm{~m}$ & $\sim 10^{-14}$ & $2.4 \times 10^{-12}$ \\
\hline $\begin{array}{c}\text { Yukawa potential } \\
U_{\text {nucl }} \sim \frac{e^{-R / \hbar}}{R}\end{array}$ & $\sim 10^{12}$ & $\approx 6 \times 10^{11}$ \\
\hline
\end{tabular}

3.2. In nano range of problems and in mesoscopics the following important issues can be determined: 1) behavior of light actinides under deep cooling as nanosized diffusion process $[15,28,34]$; 2) capability check for formation of protein nanoparticles in conditions of relatively low temperatures (e.g. in deep waters) [31, 34]; 3) search for new unknown or little-known biological nanoparticles and mesoobjects [31, 34]. Below are some qualitative considerations referring to the given questions.

3.2.1. Like a theory of fuzzy martensitic transitions developing according to diffusionless mechanism [10], the model $[15,34]$ of the $\delta$-Pu $\rightarrow \alpha$-Pu transformation, as diffusion formation of crystal particles, shows that with the decreased grain size $l$ of the initial 
phase, the temperature $T_{0}$ of the martensitic transition beginning decreases. Yet, as $l$ decreases in diffusion model the temperature $T *$ of martensitic transition finish increases and "fuzzing of temperature transformation" decreases, which is determined here as the difference $T_{0}-T_{*}$. It is connected with phonon effect (in particular, "zero" oscillations of crystal lattice). The model predicts that the reverse $\alpha-\mathrm{Pu} \rightarrow \delta$-Pu phase transformation under pulsed heating of the sample preliminary cooled down to temperatures of about $15 \mathrm{~K}$ and less is impossible because in the $\alpha$-phase particle the long-range ordering is not reached. Such particle does not have macroscopic properties and represents a mesostructure with a short-range crystal ordering.

3.2.2. Formulae of [24] for calculating average size nanoparticles in Debye approximation are overwritten as:

$$
\begin{array}{ll}
\text { small flux of embryos: } & \langle a\rangle \approx\left(\frac{75 k \theta_{D}}{8 \pi \rho}\right)^{1 / 5} t^{2 / 5}, \\
\text { large flux of embryos: } & \langle a\rangle \approx\left(\frac{27 k \theta_{D} a_{0}}{2 \cdot 6^{1 / 3} \pi^{5 / 3} \rho}\right)^{1 / 6} t^{1 / 3} .
\end{array}
$$

It has been accepted that Debye parameter $\theta_{D}$ approximately corresponds to the averaged characteristic oscillation temperature of bond expansion $(\mathrm{C}-\mathrm{C}, \mathrm{C}-\mathrm{O}, \mathrm{C}-\mathrm{N})$ of $1500 \mathrm{~K}[39]$. From formula (5) we obtain that for the time of "fast" conformational motions $10^{-10} \mathrm{~s}$ [12], in the mode of a large flux of embryos (tryptophan molecules), there can grow protein nanoparticles with the size $1.6 \mathrm{~nm}$. The found size corresponds to a globular protein (myoglobin) [12] which in considerable quantity is present in muscles of whales [45]. In the mode of a small flux of embryos, from formula (4), we obtain that in amino acid environment with the density of $\rho \sim 10^{3} \mathrm{~kg} \times \mathrm{m}^{-3}$, protein nanoparticles with the size $3.6 \mathrm{~nm}$ can grow for the same time. If we consider the growth of protein nanoparticles for the time of "slow" conformational motions $10^{-7} \mathrm{~s}$ [12], then from formula (5), in the mode of a large flux of embryos (tryptophan), we obtain that the average size of nanoparticles is $16 \mathrm{~nm}$. From formula (4), we obtain that in the mode of a small flux of embryos, protein nanoparticles with the average size about $60 \mathrm{~nm}$ can grow in amino acid environment with the density of $\rho \sim 10^{3}$

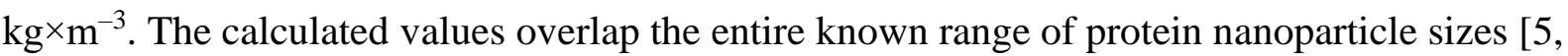
$12,38]$.

3.2.3. In the format of absolute certainty with the average density of amino acids

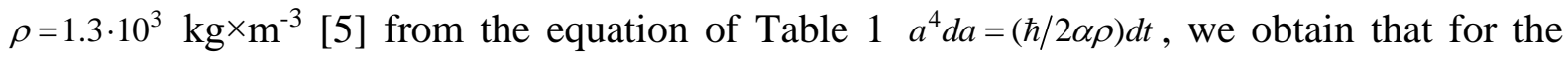
time from one million of years (conditional time after a large cosmic body that has brought amino acids in it, strikes the Earth surface) to approximately 5 billion of years (Earth age [46]), mesoobjects with the sizes from $\approx 30 \mu \mathrm{m}$ to $\approx 140 \mu \mathrm{m}$ can grow. These can be crystal skeletons of microorganisms (bacteria) formed in out-of-the-way places (glaciers, caves, extinct volcanoes, deep waters and so on).

Organic-silicon mesoobjects [47] with $\rho=0.93 \cdot 10^{3} \mathrm{~kg} \times \mathrm{m}^{-3}$ can have the related sizes. The basis of these mesoobjects is siloxane sceleton - the chain of alternate Si and $\mathrm{O}$ atoms. Elementary portion of such polymer chain consists of two adjacent atoms $\mathrm{Si}$ and attached atoms $\mathrm{C}, \mathrm{H}, \mathrm{O}$. Replacement of some $\mathrm{H}$ atoms by $\mathrm{N}, \mathrm{F}, \mathrm{S}$, and $\mathrm{Fe}$ atoms results in analogy with biological polypeptide nanochains [5]. Such analogy expands a range of questions related to the study of life as a nanoscale phenomenon [38].

Based on the data on atomic radii and the lengths of interatomic bonds [27], it is possible to evaluate an embryo size of a rubber polymer as $a_{0} \cong 0.4 \mathrm{~nm}$. Resulting from the mentioned value of average density of organic-silicon components, we will determine the mass of the least sphere-shaped embryo as $m_{0} \cong(\pi / 6) \rho a_{0}^{3} \cong 3.09 \cdot 10^{-26} \mathrm{~kg}$. Then, under the principle of uncertainty according to the Table 1 formula, one can evaluate a typical size of a globular organic-silicon nanocrystal: 


$$
a_{\max }^{u n} \approx \frac{2 m_{0} a_{0}^{3}}{9 \hbar \Delta t_{\min }} \cong 60 \mathrm{~nm},
$$

where $\Delta t_{\min }=1 / \omega_{e} c=7 \cdot 10^{-14} \mathrm{~s}\left(\omega_{e} \cong 10^{5} \mathrm{~m}^{-1}\right.$ [27] $)$. This size in a scale of magnitude corresponds to the sizes of biological equivalents (ribosomes) [5]. If similar to translational crystal symmetry, we accept $a_{\text {trans }}=2 a_{0} \cong 0.8 \mathrm{~nm}$ as an embryo size, then the evaluated typical size will increase a factor of $2^{6}=64$ :

$$
a_{\max }^{u n} \approx \frac{2 m_{0} a_{\text {trans }}^{3}}{9 \hbar \Delta t_{\text {min }}} \cong 3.84 \mu \mathrm{m} .
$$

This size in a scale of magnitude corresponds to the sizes of biological mesoobjects [5] and protozoa (archaea) [48]. The calculated sizes can be significantly increased, if we substitute a part of $\mathrm{H}$ atoms by significantly heavier atoms contained in standard biological organisms. The issue of existence of such hypothetical biological structures, containing silicon, remains open. It looks reasonable to search for the mentioned structures in places, where rubber-bearing plants grow.

3.3. With reference to unobservable cosmological objects, cosmic sphere [30] with $a_{c} \cong 9 \times 10^{27} \mathrm{~m}$ and the calculated above maximal cosmological object with $a_{\max }^{c} \cong 6 \times 10^{27} \mathrm{~m}$ are not considered by cosmological standard models $[1,6]$.

\section{Conclusions}

Under the proposed kinetic approach, the uncertainty principle covers a wider spectrum of object formation processes than the absolute certainty principle. It especially refers to nano-range-of-problems, mesoscopics, and to cosmology.

Both principles mutually complement each other and predict formation of objects that so far are not widely known or, at least, well described in scientific literature.

\section{$* * *$}

1. Penrose, R. The Road to Reality. A Complete Guide to the Laws of the Universe. Jonathan Cape, London (2004)

2. Kadomtsev, B.B. Dynamics and Information. Soviet Physics Uspekhi, 37(5), 425499 (1994)

3. Von Oppen, G. (1994) Objects and Environment. Physics Uspekhi, 39(6), 617-620.

4. Auffray, J.-P. Quantum Meteorites: An Extemporaneous Description of the System of the World. Journal of Modern Physics, 6(8), 878-889 (2015)

5. Pool, C.P. and Owens, F.J. Introduction to Nanotechnology. John Wiley \& Sons, Lnc., London (2003)

6. Lukash, V.N., Mikheeva, E.V., and Malinovsky, A.M. Formation of the Large-Scale Structure of the Universe. Physics-Uspekhi, 54(10),1017-1040 (2011)

7. Perl, M.L. Certainty and Uncertainty in the Practice of Science: Electrons, Muons, and Taus. SLAC-PUB8010-REV (1999)

8. Hellen, E. K. and Alava, M. J. Persistence in cluster-cluster aggregation. Physical Reviev E, 66(26), 026120-1 - 026120-9 (2002)

9. Slyadnikov, E.E. Two-Level Quantum System in a Crystal under Deformation (in Russian). Physical Mesomechanics, 6(3), 23-28 (2003)

10. Malygin, G.A. Nanoscope Size Effects on Martensitic Transformation in Shape Memory Alloys. Physics of the Solid State, 50, 1538-1543 (2008)

11. Vanossi, A., Manini, N., Urbakh, M., Zapetti, S., and Tosatti, E. Modeling Friction: From Nanoscale to Mesoscale. Review of Modern Physics, 85(2), 529-562 (2013)

12. Suzdalev, I.P. Nanotechnology: Physics-Chemistry of Nanoparticles, Nanostructures and Nanomaterials (in Russian). Synergetics: From the Past to Future, Komkniga, Moscow (2006)

13. Morokhov, I.D., Petinov, V.P., Turusov, L.P., and Petrunin, V.F. Structure and Properties of Fine Metallic Particles. Soviet Physics Uspekhi, 24, 295-334 (1981)

14. Lin, E.E. Cluster Model of Formation of Subnuclear and Subatomic Objects. Journal of Modern Physics, 5(3), 107-111 (2014)

15. Lin, E.E. Mesokinetics of Growth of Nanostructured Objects with Strong Interatomic Bonds. In: J. Taylor, Ed., Advances in Chemistry Research, 5, 171-190. Nova Science Publishers, New York (2010)

16. Persico, E. Fundamentals of Quantum Mechanics. Prentice-Hall (1963)

17. Man'ko, V.I. Coherent State (in Russian). In: A. M. Prokhorov, Ed., Physical Encyclopedia, Soviet Encyclopedia, Moscow, 2, 392-394 (1990) 
18. Wagner, P. and Zhong, Y.M. Cluster Formation in Disordered Systems and Nuclear Fragmentation. Nuclear Physics A, 592, 385-412 (1995)

19. Okun, L.B. Current Status of Elementary Particle Physics. Physics-Uspekhi, 41, 553-557 (1998)

20. Dremin, I.M. The Quark-Gluon Medium. Physics-Uspekhi, 53, 123-1149 (2010)

21. Lozovik, Y.E. and Popov, A.M. Formation and Growth of Carbon Nanostructures: Fullerens, Nanoparticles, Nanotubes and Cones. Physics Uspekhi, 40, 717-737 (1997)

22. Mukhin, K.N. and Patarakin, O.O. Exotic Processes in Nuclear Physics. Physics-Uspekhi, 43, 799-839 (2000)

23. Panov, I.V. and Thielemann, F.K. Fission and the r-Process: Competition between Neutron-Induced and Beta-Delayed Fission. Astronomic Letters, 30, 647-655 (2004)

24. Lin, E.E. Stochastic Mechanism of Formation of Mutational Protein Nanoparticles and Mesoobjects. Curr Trends Biomedical Eng \& Biosci; 18(3): 001-005 (2019)

25. Vol'kenstein, M.V. Molecular biophysics (inRussian). Moscow: "Nauka" (1975)

26. Zbarskii, I.B. Proteins (in Russian). Big Soviet Encyclopaedia. Moscow: SovietEncyclopaedia. 3: 317-323 (1970)

27. Ravdel', A.A, Ponomareva, A.M. (Ed.). Handbook of physical-chemical values (in Russian). Leningrad: Khimia (1983)

28. Lin, E.E. Kinetics of Formation of Objects Revealing Quantum Properties. Scientific Research Publishing, Inc. Wuhan (2016)

29. Teerikorpi P. et al. The Envolving Universe and the Origin of Life. The Search for Our Cosmic Roots. Springer-Science (2008)

30. Lin, E.E. On Boundaries of Cosmos. World Journal of Mechanics, 5, 1-6 (2015)

31. Lin, E. E. Revealing the Uncertainty and Absolute Certainty Principles in the Kinetics of Objects Formation. World Journal of Mechanics, 8(4), 82-93 2018

32. Matson, L. On Durk Matter Identification. World Journal of Mechanics, 7(1), 133-141 (2017)

33. Ishanov, B.S., Kapitonov, I.M. and Yudin, N.P. Particles and Atomic Nuclei (In Russian). LKI, Moscow (2007)

34. Lin E. E. Asymptotic Kinetics of Formation of Objects with Quantum Properties (In Russian). RFNCVNIIEF, Sarov (2019).

35. Selinov, I.P. Atomic Nuclei: Structure and Systematization (in Russian). Nauka, Moscow (1990)

36. Tarasov, V.N., Gridnev K.A., Grainer, W., Schramm, S., Gridnev, D.K., Tarasov, D.V., Vinas, X. Peninsula of Neutron Stability of Nuclei in the Neighbourhood of Neutron Magic Number $N=126$. Bulletin of the Russian Academy of Sciences. Physics, 77(7), 842-848 (2013)

37. Shenderova, O.A., Zhinov, V.V. and Brunner, D.W. Carbon Nanostructures. Critical Reviews in Solid State and Material Sciences, 27(3), 227-356 (2002)

38. Mann, S. Life as a Nanoscale Phenomenon. Angew. Chem. Int. Ed., 47, 5306-5320 (2008)

39. Syue-sen', T. Physical Mechanics. (Translated from Chinese) Mir, Moscow (1965)

40. Myosin. Big Soviet Encyclopaedia, Moscow: Soviet Encyclopaedia, 16, 901-902 (1974) (In Russian).

41. Neurofibriles. Big Soviet Encyclopaedia, Moscow: Soviet Encyclopaedia, 17, 1244 (1974) (In Russian).

42. Bartini, R.O. Relations between Physical Constants. Progress in Physics, 3, 34-40 (2005)

43. Utrobin, V.P. Supernovae. In: Prokhorov, A.M., Ed., Physical Encyclopedia, 4, 433-435, Big Russian Encyclopedia, Moscow (1994) (In Russian).

44. Lyubarskii, Yu.E. and Syunyaev, R.A. Astronomy and Astrophysics. In: Grigor'ev, S. and Meilikhov, E.Z., Eds., Physical Quantities Handbook, 1197-1230, Energoatomizdat, Moscow (1991) (In Russian).

45. Whales. In: Big Soviet Encyclopaedia, Moscow: Soviet Encyclopedia, 12, 735-737 (1973) (In Russian).

46. Kozenko, A.V. Earth. In: Prokhorov, A.M., Ed., Physical Encyclopedia, 2, 78. Big Russian Encyclopedia, Moscow (1990) (In Russian).

47. Asnovitch, E.Z., Petrashko, A.I. Silicon-organic Compounds. In: Vvedenskii B.A. and Vul B.M., Ed., Physical Encyclopedia, 2, 513-517. Soviet Encyclopedia, Moscow (1962) (In Rusian).

48. Krieg Noel Bergey's Manual of Systematic Bacteriology. US: Springer, 21 -26 (2005)

\section{Perepelkin E.E., Polyakova R.V., Kovalenko A.D., Tarelkin A.A. The Boundary Value Problem for Elliptic Equation in the Corner Domain in the Numerical Simulation of Magnetic Systems}

doi: 10.18411/scienceconf-03-2021-03

idsp: scienceconf-03-2021-03

\section{Abstract}

The formulation of the boundary value problem arises in magnitostatics when finding the magnetic field distribution by the method of two scalar potentials in the domain comprising ferromagnetic and vacuum. The problem nonlinearity is stipulated by the dependence 